\title{
Information Policy in Contests with Little Noise
}

\author{
Pouya Azimi Garakani, Oliver Gürtler \\ Department of Economics, University of Cologne, Albertus-Magnus-Platz, Cologne, Germany \\ E-mail: pouya.azimi@gmail.com,oliver.guertler@uni-koeln.de \\ Received August 15, 2011; revised September 12, 2011; accepted September 22, 2011
}

\begin{abstract}
In this paper, we analyze a contest organizer's decision to release intermediate information to the contestants. Contrary to the existing literature, we assume that there is little noise affecting the contest outcome so that there may be only mixed-strategy equilibria at the contest stage. Optimal information policy is found to depend on the prize spread and information may be revealed if contestants are very heterogeneous. This is different from results in the previous literature, which finds that the optimal information policy is independent of the prize structure and that information should be concealed from the contestants in case they are heterogeneous.
\end{abstract}

Keywords: Information Policy, Contest, Mixed-Strategy Equilibrium

\section{Introduction}

For a long time economists have recognized that people can affect other peoples' behavior by strategically releasing information. Spence [1], for example, has shown that job applicants can induce employers to make better wage offers by providing (favorable) information about their own education. Crawford and Sobel [2] have demonstrated that a decision-maker takes (unverifiable) information provided by another person into account if preferences of the two persons are not too dissimilar.

Recently, this idea has found its way into the study of contests or tournaments. A contest describes a situation, in which several contestants compete for prizes that have been fixed in advance. Examples abound. Rent seeking, promotions in internal labor markets, R \& D races, litigation and many more economic settings can be modeled as a contest. The contest organizer often receives information that the contestants do not have. Take the example of a contest for promotion to a higher layer in the hierarchy. The task of the contest organizer is to evaluate the contestants and to select a winner that gets promoted. If the contest lasts for a considerable period of time, the organizer collects intermediate performance information during the contest. Moreover, he may just like some of the contestants, while he dislikes others, generating inequality between the contestants. Finally, he may be in a relatively better position to assess whether the skills of

${ }^{1}$ See, for example, Ederer [3], Gürtler and Harbring [4] and Goltsman and Mukherjee [5]. the contestants fit well to the tasks they have to conduct (in particular, if the contestants are young and have just entered the labor market). No matter what interpretation one chooses, the contest organizer has information that the contestants do not have. She may thus decide to strategically reveal or conceal this information to affect contestants' actions. A couple of economic papers have analyzed how the contest organizer's optimal information policy should look like. ${ }^{1}$ One finding of these papers is that the contest organizer tends to conceal information when one of the contestants is heavily favored over the other (e.g., because of different intermediate performance ratings). If information was released to the contestants in this case, competition would be low because the trailing player understands that he has little chance to overcome the advantage of his opponent. Hence, the organizer prefers concealing information in this case. Another finding is that information policy may depend on the specific form of the contestants' effort cost function, but not on the structure of prizes.

In the contest or tournament literature it is well known that contestants' behavior is structurally different depending on whether the contest outcome is affected by lots of (random) noise or whether noise is relatively unimportant. In the former case, contestants choose pure strategies in the contest (see Lazear and Rosen [6] and Gürtler [7]), whereas there are only (nondegenerate) mixed-strategy equilibria in the latter case (see Baye et al. [8]). The literature on information policy in contests has been restrictive in so far that only the first type of contest 
has been considered. The aim of the current note is to analyze whether the findings of the literature carry over to the second type of contest. Considering a simple contest with discrete effort choices, we find that this is not true. For instance, we find that the optimal information policy may well depend on the prize spread. If the contest organizer leaves the contestants uncertain about their initial heterogeneity, there is typically a pure-strategy equilibrium where the contestants either choose low or high effort for sure, depending on the prize spread. ${ }^{2}$ If information is revealed to the contestants and they get to know that they are heterogeneous, there is a mixedstrategy equilibrium where each contestant chooses low and high effort with positive probability. Hence, depending on whether or not contestants are expected to choose high effort in the first situation (which in turn depends on the prize spread), concealing or revealing information is the preferred option. Related to this result we find that the contest organizer sometimes prefers to reveal information to the contestants if they are very heterogeneous. As indicated before, this is because they may choose low effort for sure when they do not receive any information.

\section{Description of the Model and Notation}

A principal $P$ organizes a contest between two agents $A$ and $B$. All parties are risk-neutral. The winner of the contest receives the winner prize $w_{1}$, while the loser gets $w_{2}<w_{1}$. Prizes are exogenously given. Denote by $\Delta w:=w_{1}-w_{2}$ the prize spread. The agent with the higher performance wins the contest. If both perform equally well, a fair coin determines the winner. Agent $\mathrm{i}$ 's performance $(\mathrm{i}=A, B)$ is given by

$$
y_{\mathrm{i}}=\mathrm{e}_{\mathrm{i}}+\theta_{\mathrm{i}}
$$

The variable $e_{i} \in\{0,1\}$ denotes the agent's effort. Effort is costly and costs are defined as $c_{\mathrm{i}}\left(\mathrm{e}_{\mathrm{i}}=0\right)=0$ and $c_{\mathrm{i}}\left(\mathrm{e}_{\mathrm{i}}=1\right)=c \in\left(0, \frac{\Delta w}{2}\right) . \theta_{\mathrm{i}}$ can be interpreted in

\footnotetext{
${ }^{2}$ Hence, uncertainty imposed by the contest organizer has a similar effect as noise in that both may induce the contestants to play a pure strategy.

${ }^{3}$ Note that only the difference between $\theta_{A}$ and $\theta_{B}$ has an effect on the agents' behavior. Hence, in the second state, we may also assume $\left\{\theta_{A}, \theta_{B}\right\}=\{1,1\}$.

${ }^{4}$ Note that the contest outcome is not affected by another random variable in addition to $\theta_{A}$ and $\theta_{B}$. Hence, if the agents get to know these two variables, there is no uncertainty. The model results would continue to hold if the contest outcome was affected by another noise term, as long as the influence of this noise term on the outcome was sufficiently low.

${ }^{5}$ A possible reason could be that $P$ cannot prevent the agents from sharing information.
}

different ways. For instance, it can describe an agent's previous performances in the contest. Alternatively, it may be used to describe a situation where the principal favors one agent over the other (if $\theta_{A} \neq \theta_{B}$ ). Finally, it may account for ability differences.

Under either interpretation, the principal should have superior information concerning the realization of $\theta_{\mathrm{i}}$. This is because she typically gathers the performance information, knows her preferences for the agents and may be in a better position to judge, whether the agents are able to handle certain tasks. To account for this, we assume $\theta_{\mathrm{i}}$ to be a random variable and the principal the only one to observe its realization (before agents choose their efforts). More concretely, we assume three states of nature. We either have $\Theta_{1}=\left\{\theta_{A}, \theta_{B}\right\}=\{1,0\}, \Theta_{2}=\{0,0\}$ or $\Theta_{3}=\{0,1\}$. This means that each agent can be in the leading position, but also that they may tie before choosing their efforts ${ }^{3}$. The states occur with probability $p_{1}$, $p_{2}$ and $p_{3}$, respectively. At the outset, the agents are homogeneous and, thus, $p_{1}=p_{3}$.

While the principal privately observes the state of nature, she can reveal her information to the agents. ${ }^{4}$ In this context, we assume that she cannot misrepresent the information, i.e., she cannot lie about the state of nature. For instance, if performance information is recorded within a firm, the principal may withhold the records from the agents, but she cannot forge them. Moreover, the principal is restricted in that she can either inform both agents about the state of nature or none of them. ${ }^{5}$

Summarizing, a strategy for the principal consists of a triple $s_{p}=\left(I_{1}, I_{2}, I_{3}\right)$, where $I_{j}(j=1,2,3)$ is a variable indicating whether the principal reveals her information to the agents $\left(I_{j}=1\right)$ in state $j$ or not $\left(I_{j}=0\right)$. In total, the principal has $2^{3}=8$ different (pure) strategies some of which lead to the same outcome (see the analysis in Section 3). We assume that the principal cannot commit to a strategy, but chooses the revelation policy that is optimal for her ex post, i.e., after the state of nature has been realized. The agents' strategies can be written as a quadruple $s_{A}=\left(q_{1}, q_{2}, q_{3}, q_{n}\right)$, with $q_{j}$ as the agent's effort choice if he is told to be in state $j$ and $q_{n}$ as his effort if the principal withholds her information from the agents.

The principal chooses her strategy so as to maximize the sum of expected efforts. The agents choose their strategies in order to maximize the expected payment minus costs entailed by effort.

\section{Model Solution}

When agents decide about their effort, they trade-off the higher effort costs from a positive effort with the in- 
creased probability of receiving the winner prize. Depending on which is higher, they choose an effort of 1 , an effort of 0 or they mix between these two alternatives. If agents know which state they are in, they are able to calculate the increase in winning-probability that a high effort entails. This, however, is no longer ensured if the principal keeps information secret and agents do not know what the relevant state is. Here, agents use Bayes' rule to update their belief about the state. The updating process, in turn, depends on the strategy that the agents expect the principal to play. This means that we cannot separate the optimal choice of the agents from that of the principal. Hence, in the following lemmas we characterize agents' optimal efforts for different strategies of the principal. ${ }^{6}$

Lemma 1 If the principal plays $(1,1,1),(1,1,0)$, $(1,0,1)$ or $(0,1,1)$, the agents always correctly infer what the relevant state is. If agent $\mathrm{i}$ believes to be in state 2 , he chooses $\mathrm{e}_{\mathrm{i}}=1$. Otherwise, he chooses $\mathrm{e}_{\mathrm{i}}=1$ with probability $r_{l}=\frac{\Delta w-2 c}{\Delta w}$ (if he believes to be leading by 1 ) or $\mathrm{e}_{\mathrm{i}}=0$ with probability $r_{t}=\frac{2 c}{\Delta w}$ (if he believes to be trailing by 1 ).

Lemma 1 characterizes the agents' behavior if they know which state they are in. If there is a tie, both have realistic chances of winning and, therefore, both choose high effort for sure. If one agent is ahead of the other, in contrast, there is no longer a pure-strategy equilibrium. This is because the leading agent can secure himself a win by choosing high effort. This would discourage the trailing agent who would choose zero effort and drop out of the contest. Then, however, the leading agent need no longer bear the cost of a positive effort since zero effort would suffice to win the contest Yet, if the leading agent chooses zero effort, the trailing agent might find it beneficial to choose high effort to catch up with his opponent and so on. Summarizing, unequal starting positions lower competition. Agents choose low and high effort with strictly positive probability.

Lemma 2 If the principal plays $(0,1,0)$, the agents' only information is whether or not they are in state 2. If agent $\mathrm{i}$ is told to be in state 2 , he chooses $\mathrm{e}_{\mathrm{i}}=1$. Otherwise, he chooses $\mathrm{e}_{\mathrm{i}}=1$ if $\Delta w>4 c$ and $\mathrm{e}_{\mathrm{i}}=0$ if $\Delta w<4 c$ (for $\Delta w=4 c$ both effort levels lead to the same payoff).

Lemma 2 shows that the strategy of the principal (i.e., the information policy) can have significant effects on the agents' efforts. More concretely, we see that there exists no longer a (non-generic) mixed-strategy equilib-

\footnotetext{
${ }^{6}$ All formal proofs are available from the authors upon request.
}

rium once the principal reveals information only in state 2. The intuition is as follows: The mixed-strategy equilibrium resulted from the fact that, in case the state is commonly known and agents do not tie, the leading agent could ensure himself a win by choosing positive effort, which would completely discourage the trailing agent. If the principal plays $(0,1,0)$, however, agents do not know whether they are leading or trailing behind. In this case, choosing a positive effort increases the winning-probability in at least one state of nature (regardless of the opponent's effort) and, hence, the expected winning-probability. As a result, we have an equilibrium in pure strategies. If the prize spread is relatively high, both agents choose high effort. This means that concealing information may increase expected efforts by leaving the contestants uncertain on whether they are in the leading position or trailing behind. To get unambiguous results, we assume in the following that both agents choose the high effort in case $\Delta w=4 c$.

Lemma 3 If the principal plays $(0,0,0)$, the agents do not get any information about the realized state.

Agent $\mathrm{i}$ chooses $\mathrm{e}_{\mathrm{i}}=1$ if $\Delta \mathrm{w}>\frac{2}{1-p_{1}} c$ and $\mathrm{e}_{\mathrm{i}}=0$ if $\Delta w<\frac{2}{1-p_{1}} c$ (for $\Delta w=\frac{2}{1-p_{1}} c$ both effort levels lead to the same payoff).

Lemma 3 is in spirit similar to Lemma 2. It implies that a mixed-strategy equilibrium does not exist if the agents do not know which state they are in. Again, concealing information may lead the agents to choose the high effort for sure and, hence, may increase expected effort. Again, we assume both agents to choose the high effort in case

$$
\Delta w=\frac{2}{1-p_{1}} c .
$$

Lemma 4 If the principal plays $(1,0,0)$, the agents' only information is whether or not they are in state 1 . If agent $A(B)$ is told to be in state 1 , he chooses $\mathrm{e}_{A}=1$ $\left(\mathrm{e}_{B}=1\right)$ with probability $r_{A}=\frac{\Delta w-2 c}{\Delta w}\left(r_{B}=\frac{2 c}{\Delta w}\right)$. If not, optimal efforts depend on the prize spread. If $\Delta w>$ $\frac{2\left(1-p_{1}\right)}{\left(1-2 p_{1}\right)} c$, both agents choose the high effort for sure. If $\Delta w=\frac{2\left(1-p_{1}\right)}{\left(1-2 p_{1}\right)} c, \quad B$ chooses the high effort for sure, while A mixes between the two effort levels. If $\Delta w<$ $\frac{2\left(1-p_{1}\right)}{\left(1-2 p_{1}\right)} c, A(B)$ chooses $\mathrm{e}_{A}=1\left(\mathrm{e}_{B}=1\right)$ with proba- 
bility

$r_{A}=\frac{2\left(1-p_{1}\right) c-\left(1-2 p_{1}\right) \Delta w}{p_{1} \Delta w} \quad\left(r_{B}=\frac{\left(1-p_{1}\right)(\Delta w-2 c)}{p_{1} \Delta w}\right)$.

Lemma 4 combines the ideas from the previous lemmas. The principal informs the agents in state 1 , in which case they play a mixed-strategy equilibrium. In the other states, she conceals her information. By doing this, she may induce both agents to choose the high effort for sure. Note that the analysis is completely analogous for the principal's strategy $(0,0,1)$.

Having analyzed the consequences of the principal's strategies, we are now able to characterize her behavior. This is done in the following proposition: ${ }^{7}$

Proposition 1 If $\Delta w \geq 4 c$, the principal either plays $(0,0,0)$ or $(0,1,0)$. If $\Delta w \in\left[\frac{2}{1-p_{1}} c, 4 c\right)$, she plays $(0,0,0)$. Finally, if $\Delta w<\frac{2}{1-p_{1}} c$, she plays $(1,1,1)$, $(1,1,0)$ or $(0,1,1)$

Proposition 1 states that $P$ often wishes to conceal the information from the agents. This is intuitive since we have seen before that agents often choose higher efforts in case they do not learn whether they are in the leading position or not. Only for a very low prize spread it is optimal for the principal to always reveal the state of nature (either directly or indirectly). If the prize spread is low, concealing information induces the agents to choose zero effort. Then, of course, $P$ prefers to reveal her information. Contrary to the previous literature on feedback in contests we therefore find that optimal information policy and optimal prize structure may be interdependent and should not be determined in isolation. Moreover, we see that $P$ sometimes prefers to reveal information even if agents are heterogeneous. As indicated in the introduction, this finding again differs from findings in the existing literature.

\section{Acknowledgements}

We would like to thank an anonymous referee as well as Bernd Irlenbusch, Dirk Sliwka and participants at the research seminar in Cologne for helpful comments.

\section{References}

[1] A. M. Spence, "Job Market Signaling,” Quarterly Journal of Economics, Vol. 87, No. 3, 1981, pp. 355-374. doi: $10.2307 / 1882010$

[2] V. Crawford and J. Sobel, "Strategic Information Transmission,” Econometrica, Vol. 50, No. 6, 1982, pp. 14311451. doi:10.2307/1913390

[3] F. Ederer, "Feedback and Motivation in Dynamic Tournaments,” Journal of Economics and Management Strategy, Vol. 19, No. 3, 2010, pp. 733-769. doi:10.1111/j.1530-9134.2010.00268.x

[4] O. Gürtler and C. Harbring, "Feedback in Tournaments under Commitment Problems: Experimental Evidence," Journal of Economics and Management Strategy, Vol. 19, No. 3, 2010, pp. 771-810. doi:10.1111/j.1530-9134.2010.00269.x

[5] M. Goltsman and A. Mukherjee, "Interim Performance Feedback in Multistage Tournaments: The Optimality of Partial Disclosure," Journal of Labor Economics, Vol. 29, No. 2, 2011, pp. 229-265. doi:10.1086/656669

[6] E.P. Lazear and S. Rosen, "Rank-Order Tournaments as Optimum Labor Contracts," Journal of Political Economy, Vol. 89, No. 5, 1981, pp. 841-864. doi:10.1086/261010

[7] O. Gürtler, "The First-Order Approach in Rank-Order Tournaments,” Economics Letters, Vol. 111, No.3, 2011, pp. 185-187. doi:10.1016/j.econlet.2011.02.013

[8] M. R. Baye, D. Kovenock and C. G. de Vries, "The AllPay Auction with Complete Information,” Economic Theory, Vol. 8, No. 2, 1996, pp. 291-305.

\footnotetext{
${ }^{7}$ The proof of the proposition proceeds in two steps. First, we analyze which strategies lead to the highest payoff for the principal, i.e., the highest expected value for the aggregate efforts. Second, we analyze whether the principal has an incentive to deviate from these strategies. Not all strategies are robust to deviations. Suppose $P$ plays $(1,0,1)$ in which case the agents infer to be in state 2 if they do not get informed about the state of nature. Then, in states 1 and $3 P$ would also want to conceal information in order to make the agents think that they are in state 2 . A similar problem arises with the strategy $(1,1,1)$, at least for certain specifications of the off-equilibrium beliefs. Still, there exist off-equilibrium beliefs sustaining $(1,1,1)$ as an equilibrium, for example if agents believe that they are in state 1 in case information is withheld.
} 